\title{
ADDITIVE FUNCTIONAL EQUATION WITH SEVERAL VARIABLES AND ITS STABILITY
}

\author{
HARK-MAHN KIM* AND Hwan-Yong SHIN**
}

\begin{abstract}
In this paper, we prove the generalized Hyers-Ulam stability of an $n$-dimensional additive functional equation, and then apply stability results to Banach modules over a unital Banach algebras.
\end{abstract}

\section{Introduction}

The stability problem of functional equations originated from a question of S. M. Ulam [8] concerning the stability of group homomorphisms.

Let $G_{1}$ be a group and $G_{2}$ a metric group with metric $\rho(\cdot, \cdot)$. Given $\epsilon>$ 0 , does there exist a number $\delta>0$ such that if a mapping $f: G_{1} \rightarrow G_{2}$ satisfies $\rho(f(x y), f(x) f(y))<\delta$ for all $x, y \in G_{1}$, then a homomorphism $h: G_{1} \rightarrow G_{2}$ exists near $f$ with $\rho(f(x), h(x))<\epsilon$ for all $x \in G_{1}$ ?

In 1941, D. H. Hyers [5] considered the case of approximately additive mappings between Banach spaces and proved the following result. Suppose that $E_{1}$ and $E_{2}$ are Banach spaces and a mapping $f: E_{1} \rightarrow E_{2}$ satisfies the following condition: if there is a number $\epsilon \geq 0$ such that

$$
\|f(x+y)-f(x)-f(y)\| \leq \epsilon
$$

for all $x, y \in E_{1}$, then the limit $h(x)=\lim _{n \rightarrow \infty} \frac{f\left(2^{n} x\right)}{2^{n}}$ exists for all $x \in E_{1}$ and there exists a unique additive mapping $h: E_{1} \rightarrow E_{2}$ such that

$$
\|f(x)-h(x)\| \leq \epsilon
$$

Received December 18, 2013; Accepted June 30, 2014.

2010 Mathematics Subject Classification: Primary 39B82, 39B52.

Key words and phrases: Hyers-Ulam stability, Banach module, B-linear.

Correspondence should be addressed to Hwan-Yong Shin, hyshin31@cnu.ac.kr.

This research was supported by Basic Science Research Program through the National Research Foundation of Korea (NRF) funded by the Ministry of Education (No.2012R1A1A2008139). 
for all $x \in E_{1}$. Moreover, if $f(t x)$ is continuous in $t \in \mathbb{R}$ for each $x \in E_{1}$, then the mapping $h$ is $\mathbb{R}$-linear.

This result was generalized by T. Aoki [2] for additive mappings and by Th. M. Rassias [7] for linear mappings by establishing an unbounded Cauchy difference. A generalization of the Th.M. Rassias theorem was obtained by P. Găvruta [4] by replacing the unbounded Cauchy difference by a general control function. The stability problem of various functional equations has been studied by a number of authors since then. Recently, P. Nakmahachalasint [6] considered the following $n$ dimensional additive functional equation

$$
f\left(\sum_{i=1}^{n} x_{i}\right)=\sum_{i=1}^{n} f\left(x_{i}\right)+\sum_{i=1}^{n} f\left(x_{i}-x_{i-1}\right),
$$

where $x_{0}:=x_{n}$ and $n \geq 2$, and then investigated its Hyers-UlamRassias stability.

In this paper, we establish the general solution of the following $n$ dimensional additive functional equation

$$
f\left(\sum_{i=1}^{n} x_{i}\right)=\sum_{i=1}^{n} f\left(x_{i}\right)+\sum_{i \neq j} f\left(x_{i}-x_{j}\right)
$$

where $n>1$ is fixed, and then we investigate its generalized Hyers-Ulam stability.

\section{The first result of Hyers-Ulam stability}

We now present the general solution of the equation (1.2) in the class of functions between two vector spaces.

Lemma 2.1. Let $X$ and $Y$ be vector spaces. A mapping $f: X \rightarrow$ $Y$ satisfies the functional equation (1.2) if and only if it satisfies the Cauchy additive functional equation

$$
f(x+y)=f(x)+f(y)
$$

for all $x, y \in X$.

Proof. Suppose a mapping $f: X \rightarrow Y$ satisfies the Cauchy additive functional equation. Then, it is straightforward to show that

$$
f\left(\sum_{i=1}^{n} x_{i}\right)=\sum_{i=1}^{n} f\left(x_{i}\right), \quad \sum_{i \neq j} f\left(x_{i}-x_{j}\right)=\sum_{i \neq j}\left[f\left(x_{i}\right)-f\left(x_{j}\right)\right]=0 .
$$

Hence, $f$ satisfies the equation (1.2). 
Suppose a mapping $f: X \rightarrow Y$ satisfies the equation (1.2). Then, by setting $x_{1}=\cdots=x_{n}:=0$ in (1.2), we can see that $f(0)=0$. Letting $x_{1}:=x$ and $x_{2}=\cdots=x_{n}:=0$ in (1.2), we have

$$
0=(n-1)[(f(x)+f(-x)],
$$

which shows that $f$ is odd. Putting $\left(x_{1}, x_{2}, \cdots, x_{n}\right):=(y, x, 0, \cdots, 0)$ in (1.2), we lead to

$$
\begin{aligned}
f(x+y)= & f(x)+f(y)+(n-2)[f(x)+f(-x)+f(y)+f(-y)] \\
& +f(y-x)+f(x-y) .
\end{aligned}
$$

By the oddness of $f$, we have $f(x+y)=f(x)+f(y)$, as desired.

From now on, we denote $X$ and $Y$ by a normed linear space and a Banach space, respectively. For simplicity, given mappings $f: X \rightarrow Y$ and $\varphi: X^{n} \rightarrow[0, \infty)$, we define a difference operator $D f$ by

$$
D f\left(x_{1}, \cdots, x_{n}\right):=\sum_{i=1}^{n} f\left(x_{i}\right)+\sum_{i \neq j} f\left(x_{i}-x_{j}\right)-f\left(\sum_{i=1}^{n} x_{i}\right)
$$

for all $x_{1}, \cdots, x_{n} \in X$, and $\Psi_{i}: X \rightarrow[0, \infty), \Phi_{i}: X \rightarrow[0, \infty)$ by

$$
\begin{aligned}
\Psi_{i}(x):= & \frac{1}{2^{i+1}}\left|n^{2}-4 n+2\right|\|f(0)\|+\frac{(n-2)}{(n-1) 2^{i}} \varphi\left(2^{i} x, 0, \cdots, 0\right) \\
& +\frac{1}{2^{i+1}} \varphi\left(2^{i} x, 2^{i} x, 0, \ldots, 0\right), \quad \forall i \geq 0, \\
\Phi_{i}(x):= & 2^{i}\left|n^{2}-4 n+2\right|\|f(0)\|+\frac{(n-2) 2^{i+1}}{(n-1)} \varphi\left(2^{-(i+1)} x, 0, \cdots, 0\right) \\
& +2^{i} \varphi\left(2^{-(i+1)} x, 2^{-(i+1)} x, 0, \cdots, 0\right), \quad \forall i \geq 0,
\end{aligned}
$$

for all $x \in X$.

Theorem 2.2. Let $\varphi: X^{n} \rightarrow[0, \infty)$ be a mapping which satisfies

$$
\sum_{i=0}^{\infty} \frac{\varphi\left(2^{i} x_{1}, \cdots, 2^{i} x_{n}\right)}{2^{i}}<\infty,\left(\sum_{i=0}^{\infty} 2^{i} \varphi\left(\frac{x_{1}}{2^{i}}, \cdots, \frac{x_{n}}{2^{i}}\right)<\infty, \text { resp. }\right)
$$

for all $x_{1}, \cdots, x_{n} \in X$. If a mapping $f: X \rightarrow Y$ satisfies the inequality

$$
\left\|D f\left(x_{1}, \cdots, x_{n}\right)\right\| \leq \varphi\left(x_{1}, \cdots, x_{n}\right)
$$

for all $x_{1}, \cdots, x_{n} \in X$, then there exists a unique additive mapping $L: X \rightarrow Y$ such that $L$ satisfies the inequality

$$
\|f(x)-L(x)\| \leq \sum_{i=0}^{\infty} \Psi_{i}(x),\left(\|f(x)-L(x)\| \leq \sum_{i=0}^{\infty} \Phi_{i}(x), \text { resp. }\right)
$$


where the mapping $L$ is given by

$$
L(x)=\lim _{n \rightarrow \infty} 2^{-m} f\left(2^{m} x\right), \quad\left(L(x)=\lim _{n \rightarrow \infty} 2^{m} f\left(2^{-m} x\right), \text { resp. }\right)
$$

for all $x \in X$. Moreover, if $f$ is measurable or $f(t x)$ is continuous in $t \in \mathbb{R}$ for each fixed $x \in X$, then the mapping $L$ is $\mathbb{R}$-linear.

Proof. If $n=2$, we set $x_{1}=x_{2}:=x$ in (2.1), then we have

$$
\begin{aligned}
\|2 f(x)-f(2 x)\| & \leq 2\|f(0)\|+\varphi(x, x) \\
& =\left|n^{2}-4 n+2\right|\|f(0)\|+2 \frac{(n-2)}{(n-1)} \varphi(x, 0)+\varphi(x, x)
\end{aligned}
$$

for all $x \in X$. If $n>2$, then we set $x_{1}:=x$ and $x_{2}=\cdots=x_{n}:=0$ in (2.1) and so we have

$$
\left\|\left(n^{2}-2 n+1\right) f(0)+(n-1)(f(x)+f(-x))\right\| \leq \varphi(x, 0, \cdots, 0)
$$

which is simplified to

$$
\|(n-1) f(0)+f(x)+f(-x)\| \leq \frac{1}{n-1} \varphi(x, 0, \cdots, 0)
$$

for all $x \in X$. Setting $x_{1}=x_{2}:=x$ and $x_{3}=\cdots=x_{n}:=0$ in (2.1), we have

$$
\begin{aligned}
& \| 2(n-2)[(n-1) f(0)+f(x)+f(-x)] \\
& -\left(n^{2}-4 n+2\right) f(0)+2 f(x)-f(2 x) \| \leq \varphi(x, x, 0, \cdots, 0)
\end{aligned}
$$

for all $x \in X$. Associating the last two inequalities, one has

$$
\begin{aligned}
\|2 f(x)-f(2 x)\| \leq & \left|n^{2}-4 n+2\right|\|f(0)\|+2 \frac{(n-2)}{(n-1)} \varphi(x, 0, \cdots, 0) \\
& +\varphi(x, x, 0, \cdots, 0)
\end{aligned}
$$

for all $x \in X$ and any fixed integer $n \geq 2$. Thus, one can prove

$$
\begin{gathered}
\left\|f(x)-2^{-m} f\left(2^{m} x\right)\right\| \leq \sum_{i=0}^{m-1}\left\|2^{-i} f\left(2^{i} x\right)-2^{-(i+1)} f\left(2^{i+1} x\right)\right\| \\
\leq \sum_{i=0}^{m-1}\left[\frac{1}{2^{i+1}}\left|n^{2}-4 n+2\right|\|f(0)\|+\frac{(n-2)}{(n-1) 2^{i}} \varphi\left(2^{i} x, 0, \cdots, 0\right)\right. \\
\left.\quad+\frac{1}{2^{i+1}} \varphi\left(2^{i} x, 2^{i} x, 0, \cdots, 0\right)\right]
\end{gathered}
$$

for all $x \in X$ and for every positive integer $m$. Therefore, for every positive integers $m$ and $k$ with $m>k$, we obtain 


$$
\begin{aligned}
\left\|2^{-k} f\left(2^{k} x\right)-2^{-m} f\left(2^{m} x\right)\right\| & =2^{-k}\left\|f\left(2^{k} x\right)-2^{-(m-k)} f\left(2^{m-k} 2^{k} x\right)\right\| \\
& \leq \sum_{i=0}^{m-k-1} 2^{-k} \Psi_{i}\left(2^{k} x\right)=\sum_{i=k}^{m-1} \Psi_{i}(x)
\end{aligned}
$$

for all $x \in X$. Since $\sum_{i=0}^{\infty} \Psi_{i}(x)<\infty$ and $\sum_{i=k}^{m-1} \Psi_{i}(x) \rightarrow 0$ as $k \rightarrow \infty$, the sequence $\left\{2^{-m} f\left(2^{m} x\right)\right\}$ is a Cauchy in the complete normed space $Y$. Thus, we may define

$$
L(x):=\lim _{m \rightarrow \infty} 2^{-m} f\left(2^{m} x\right), \quad \forall x \in X .
$$

Letting $m \rightarrow \infty$ in (2.3), then we get the inequality (2.2). Replace $\left(x_{1}, \cdots, x_{n}\right)$ by $\left(2^{m} x_{1}, \cdots, 2^{m} x_{n}\right)$ in $(2.1)$ and divide it by $2^{m}$. Taking the limit in the resulting inequality, we see that

$$
L\left(\sum_{i=1}^{n} x_{i}\right)=\sum_{i=1}^{n} L\left(x_{i}\right)+\sum_{i \neq j} L\left(x_{i}-x_{j}\right)
$$

for all $x_{1}, \cdots, x_{n} \in X$. By Lemma 2.1, the mapping $L$ is additive. Under the assumption that $f$ is measurable or $f(t x)$ is continuous in $t \in \mathbb{R}$ for all $x \in X$, by the same reasoning as in the proof of [7], the additive mapping $L: X \rightarrow Y$ satisfies

$$
L(t x)=t L(x), \quad \forall x \in X, \forall t \in \mathbb{R} .
$$

That is, $L$ is $\mathbb{R}$-linear.

Now, we finally prove the uniqueness. Let $L^{\prime}: X \rightarrow Y$ be another additive mapping satisfying (2.2). Then we have

$$
\begin{aligned}
\left\|L(x)-L^{\prime}(x)\right\| & =\frac{1}{2^{m}}\left\|L\left(2^{m} x\right)-L^{\prime}\left(2^{m} x\right)\right\| \\
& \leq \frac{1}{2^{m}}\left(\left\|L\left(2^{m} x\right)-f\left(2^{m} x\right)\right\|+\left\|f\left(2^{m} x\right)-L^{\prime}\left(2^{m} x\right)\right\|\right) \\
& \leq 2 \sum_{i=0}^{\infty} \frac{\Psi_{i}\left(2^{m} x\right)}{2^{m}}=2 \sum_{i=m}^{\infty} \Psi_{i}(x)
\end{aligned}
$$

for all $x \in X$ and all $m \in \mathbb{N}$. This series converges to 0 as $m \rightarrow \infty$. So we can conclude that $L(x)=L^{\prime}(x)$ for all $x \in X$.

Corollary 2.3. Let $p \neq 1$ be a positive real number and $\theta, \delta \geq 0$ be real numbers. If a mapping $f: X \rightarrow Y$ satisfies the inequality

$$
\left\|D f\left(x_{1}, x_{2}, \cdots, x_{n}\right)\right\| \leq \delta+\theta \sum_{i=1}^{n}\left\|x_{i}\right\|^{p}
$$


for all $x_{1}, \ldots, x_{n} \in X$, where $\delta=0$ when $p>1$, then there exists a unique additive mapping $L: X \rightarrow Y$ such that $L$ satisfies the inequality

$\|f(x)-L(x)\| \leq\left|n^{2}-4 n+2\right|\|f(0)\|+\left(\frac{3 n-5}{n-1}\right) \delta+\frac{(4 n-6) 2^{p} \theta}{(n-1)\left|2-2^{p}\right|}\|x\|^{p}$

for all $x \in X$, where $f(0)=0$ if $p>1$. Moreover, if $f$ is measurable or $f(t x)$ is continuous in $t \in \mathbb{R}$ for each fixed $x \in X$, then the mapping $L$ is $\mathbb{R}$-linear.

Proof. Letting $\varphi\left(x_{1}, x_{2}, \cdots, x_{n}\right)=\delta+\theta \sum_{i=1}^{n}\left\|x_{i}\right\|^{p}$ and applying Theorem 2.2, we get the desired result, as claimed.

Corollary 2.3 leaves the case $p=1$ undecided. We remark that 1 is a critical value of $p$ to which Corollary 2.3 cannot extended. In fact, we shall show that for some $\varepsilon>0$ one can find a function $f: \mathbb{R} \rightarrow \mathbb{R}$ such that

$$
\left|D f\left(x_{1}, x_{2}, \cdots, x_{n}\right)\right| \leq \varepsilon \sum_{i=1}^{n}\left|x_{i}\right|
$$

for all $x_{1}, x_{2}, \cdots, x_{n} \in \mathbb{R}$, however, at the same time, there is no constant $\delta$ and no additive function $T: \mathbb{R} \rightarrow \mathbb{R}$ satisfying the condition

$$
|f(x)-T(x)| \leq \delta|x|
$$

for all $x \in \mathbb{R}$. The following is a modified example of Z. Gajda's example [3], which illustrates that Corollary 2.3 fails to hold for $p=1$.

Fix $\varepsilon>0$ and put $\mu:=\frac{\varepsilon}{2\left(n^{2}+1\right)}$. First, we define a function $\phi$ : $\mathbb{R} \rightarrow \mathbb{R}$ by

$$
\phi(x):=\left\{\begin{array}{lll}
\mu & \text { for } & x \in[1, \infty) \\
\mu x & \text { for } & x \in(-1,1) \\
-\mu & \text { for } \quad x \in(-\infty,-1] .
\end{array}\right.
$$

Evidently, $\phi$ is continuous and $|\phi(x)| \leq \mu$ for all $x \in \mathbb{R}$. Therefore, a function $f: \mathbb{R} \rightarrow \mathbb{R}$ may be defined by the formula

$$
f(x):=\sum_{k=0}^{\infty} \frac{\phi\left(2^{k} x\right)}{2^{k}}, \quad x \in \mathbb{R} .
$$

Since $f$ is defined by means of a uniformly convergent series of continuous functions, $f$ itself is continuous and $|f(x)| \leq \sum_{k=0}^{\infty} \frac{\mu}{2^{k}}=2 \mu$.We are going to show that $f$ satisfies the inequality (2.4). If $x_{1}=x_{2}=\cdots=x_{n}=0$, 
then (2.4) is trivially fulfilled. Next, assume that $0<\left|x_{1}\right|+\left|x_{2}\right|+\cdots+$ $\left|x_{n}\right|<1$. Then there exists an $N \in \mathbb{N}$ such that

$$
\frac{1}{2^{N}} \leq\left|x_{1}\right|+\left|x_{2}\right|+\cdots+\left|x_{n}\right|<\frac{1}{2^{N-1}} .
$$

Hence, $\left|2^{N-1} x_{i}\right|<1,\left|2^{N-1}\left(x_{i}-x_{j}\right)\right|<1$ for all $i, j=1,2, \cdots, n$ and $\left|2^{N-1}\left(x_{1}+x_{2}+\cdots+x_{n}\right)\right|<1$, which implies that for each $k \in$ $\{0,1,2, \cdots, N-1\}$ the numbers $2^{k} x_{i}, 2^{k}\left(x_{i}-x_{j}\right)$ and $2^{k}\left(x_{1}+x_{2}+\cdots+x_{n}\right)$ remain in the interval $(-1,1)$. Since $\phi$ is linear on this interval, we infer that

$$
D \phi\left(2^{k} x_{1}, 2^{k} x_{2}, \cdots, 2^{k} x_{n}\right)=0
$$

for all $k=0,1, \cdots, N-1$. As a result, we get

$$
\begin{aligned}
\frac{\left|D f\left(x_{1}, x_{2}, \cdots, x_{n}\right)\right|}{\left|x_{1}\right|+\left|x_{2}\right|+\cdots+\left|x_{n}\right|} & \leq \sum_{k=N}^{\infty} \frac{\left|D \phi\left(2^{k} x_{1}, 2^{k} x_{2}, \cdots, 2^{k} x_{n}\right)\right|}{2^{k}\left(\left|x_{1}\right|+\left|x_{2}\right|+\cdots+\left|x_{n}\right|\right)} \\
& \leq \sum_{k=0}^{\infty} \frac{\left(n^{2}+1\right) \mu}{2^{k} 2^{N}\left(\left|x_{1}\right|+\left|x_{2}\right|+\cdots+\left|x_{n}\right|\right)} \\
& \leq 2\left(n^{2}+1\right) \mu=\varepsilon .
\end{aligned}
$$

Finally, assume that $\left|x_{1}\right|+\left|x_{2}\right|+\cdots+\left|x_{n}\right| \geq 1$. Then merely by virtue of the boundedness of $f$, we have

$$
\frac{\left|D f\left(x_{1}, x_{2}, \cdots, x_{n}\right)\right|}{\left|x_{1}\right|+\left|x_{2}\right|+\cdots+\left|x_{n}\right|} \leq 2\left(n^{2}+1\right) \mu=\varepsilon .
$$

Thus we conclude that $f$ satisfies (2.4) for all $x_{1}, x_{2}, \cdots, x_{n}$.

Now, contrary to what we claim, suppose that there exist a $\delta \in[0, \infty)$ and an additive function $T: \mathbb{R} \rightarrow \mathbb{R}$ such that (2.5) holds true. Then, it follows from the continuity of $f$ that $T$ is bounded on some neighborhood of zero. Now, by a classical result (see e.g. [1], 2.1.1., Theorem 1) there exists a real constant $c$ such that

$$
T(x)=c x, \quad \forall x \in \mathbb{R} .
$$

Hence,

$$
|f(x)-c x| \leq \delta|x|, \quad \forall x \in \mathbb{R},
$$

which implies that

$$
\left|\frac{f(x)}{x}\right| \leq \delta+|c|, \quad \forall x \in \mathbb{R}-\{0\} .
$$


On the other hand, we can choose an $N^{\prime} \in \mathbb{N}$ so large that $N^{\prime} \mu>\delta+|c|$. Then picking out an $x$ from the interval $\left(0, \frac{1}{2^{N^{\prime}}-1}\right)$, we have $2^{k} x \in(0,1)$ for each $k \in\left\{0,1,2, \cdots, N^{\prime}-1\right\}$. Consequently, for this $x$ we have

$$
\frac{f(x)}{x} \geq \sum_{k=0}^{N^{\prime}-1} \mu=N^{\prime} \mu>\delta+|c|,
$$

which yields a contradiction. Thus the function $f$ provides a good example to the effect that Corollary 2.3 fails to hold for $p=1$.

\section{The second result of Hyers-Ulam stability}

In this part, we investigate alternative generalized Hyers-Ulam stability of the equation (1.2).

Theorem 3.1. If a mapping $f: X \rightarrow Y$ satisfies the inequality (2.1), and if $\varphi: X^{n} \rightarrow[0, \infty)$ satisfies

$$
\sum_{i=0}^{\infty} \frac{\varphi\left(n^{i} x_{1}, \cdots, n^{i} x_{n}\right)}{n^{i}}<\infty,\left(\sum_{i=0}^{\infty} n^{i} \varphi\left(\frac{x_{1}}{n^{i}}, \cdots, \frac{x_{n}}{n^{i}}\right)<\infty, \text { resp. }\right)
$$

for all $x_{1}, \cdots, x_{n} \in X$, then there exists a unique additive mapping $L: X \rightarrow Y$ such that $L$ satisfies the inequality

$$
\begin{gathered}
\|f(x)-L(x)\| \leq n\|f(0)\|+\sum_{i=0}^{\infty} \frac{1}{n^{i+1}} \varphi\left(n^{i} x, n^{i} x, \cdots, n^{i} x\right) \\
\left(\|f(x)-L(x)\| \leq \sum_{i=0}^{\infty} n^{i} \varphi\left(\frac{x}{n^{i+1}}, \frac{x}{n^{i+1}}, \cdots, \frac{x}{n^{i+1}}\right), \text { resp. }\right)
\end{gathered}
$$

for all $x \in X$. The mapping $L$ is given by

$$
E(x)=\lim _{m \rightarrow \infty} n^{-m} f\left(n^{m} x\right), \quad\left(E(x)=\lim _{m \rightarrow \infty} n^{m} f\left(n^{-m} x\right), \text { resp. }\right)
$$

for all $x \in X$. Moreover, if $f$ is measurable or $f(t x)$ is continuous in $t \in \mathbb{R}$ for each fixed $x \in X$, then the mapping $L$ is $\mathbb{R}$-linear.

Proof. Setting $x_{1}=\cdots=x_{n}:=x$ in (2.1), we have

$$
\|n f(x)+n(n-1) f(0)-f(n x)\| \leq \varphi(x, \cdots, x)
$$

which is simplified to

$$
\|n f(x)-f(n x)\| \leq n(n-1)\|f(0)\|+\varphi(x, \cdots, x)
$$

for all $x \in X$. Thus 


$$
\begin{aligned}
\left\|f(x)-n^{-m} f\left(n^{m} x\right)\right\| & \leq \sum_{i=0}^{m-1}\left\|\frac{f\left(n^{i} x\right)}{n^{i}}-\frac{f\left(n^{i+1} x\right)}{n^{i+1}}\right\| \\
& \leq \sum_{i=0}^{m-1}\left[\frac{n-1}{n^{i}}\|f(0)\|+\frac{1}{n^{i+1}} \varphi\left(n^{i} x, \cdots, n^{i} x\right)\right]
\end{aligned}
$$

for all $x \in X$ and all $m \geq 1$. The rest of proof is similar to the proof of Theorem 2.2.

Corollary 3.2. Let $p \neq 1$ be a positive real number and $\theta, \delta \geq 0$ be real numbers. If a mapping $f: X \rightarrow Y$ satisfies the inequality

$$
\left\|D f\left(x_{1}, \cdots, x_{n}\right)\right\| \leq \delta+\theta \sum_{i=1}^{n}\left\|x_{i}\right\|^{p}
$$

for all $x_{1}, \cdots, x_{n} \in X$, where $\delta=0$ when $p>1$, then there exists a unique additive mapping $L: X \rightarrow Y$ such that $L$ satisfies the inequality

$$
\|f(x)-L(x)\| \leq n\|f(0)\|+\frac{\delta}{n-1}+\frac{n^{p}}{\left|n-n^{p}\right|}\|x\|^{p}
$$

for all $x \in X$, where $f(0)=0$ if $p>1$. Moreover, if $f$ is measurable or $f(t x)$ is continuous in $t \in \mathbb{R}$ for each fixed $x \in X$, then the mapping $L$ is $\mathbb{R}$-linear.

\section{Applications to Banach modules}

Throughout this section, let $B$ be a unital Banach algebra with norm $|\cdot|$, and let ${ }_{B} \mathbb{B}_{1}$ and ${ }_{B} \mathbb{B}_{2}$ be left Banach $B$-modules with norms $\|\cdot\|$ and $\|\cdot\|$, respectively. A linear mapping $L:{ }_{B} \mathbb{B}_{1} \rightarrow{ }_{B} \mathbb{B}_{2}$ is called $B$-linear if

$$
L(\alpha x)=\alpha L(x)
$$

for all $\alpha \in B$ and $x \in{ }_{B} \mathbb{B}_{1}$. We denote $D_{a} f$ by

$$
D_{a} f\left(x_{1}, \cdots, x_{n}\right):=\sum_{i=1}^{n} f\left(a x_{i}\right)+\sum_{i \neq j} f\left(a x_{i}-a x_{j}\right)-a f\left(\sum_{i=1}^{n} x_{i}\right)
$$

for all $a \in B(1):=\{a \in B:|a|=1\}$ and $x_{1}, \cdots, x_{n} \in{ }_{B} \mathbb{B}_{1}$.

Theorem 4.1. Let $\varphi:{ }_{B} \mathbb{B}_{1}^{n} \rightarrow[0, \infty)$ be a mapping which satisfies

$$
\sum_{i=0}^{\infty} \frac{\varphi\left(n^{i} x_{1}, \cdots, n^{i} x_{n}\right)}{n^{i}}<\infty,\left(\sum_{i=0}^{\infty} n^{i} \varphi\left(\frac{x_{1}}{n^{i}}, \cdots, \frac{x_{n}}{n^{i}}\right)<\infty, \text { resp. }\right)
$$


for all $x_{1}, \cdots, x_{n} \in{ }_{B} \mathbb{B}_{1}$. If a mapping $f:{ }_{B} \mathbb{B}_{1} \rightarrow{ }_{B} \mathbb{B}_{2}$ satisfies the inequality

$$
\left\|D_{a} f\left(x_{1}, \cdots, x_{n}\right)\right\| \leq \varphi\left(x_{1}, \cdots, x_{n}\right)
$$

for all $a \in B(1)$ and $x_{1}, \cdots, x_{n} \in{ }_{B} \mathbb{B}_{1}$, and if $f$ is measurable or $f(t x)$ is continuous in $t \in \mathbb{R}$ for each fixed $x \in{ }_{B} \mathbb{B}_{1}$, then there exists a unique $B$-linear mapping $L:{ }_{B} \mathbb{B}_{1} \rightarrow{ }_{B} \mathbb{B}_{2}$ such that $L$ satisfies the inequality

$$
\begin{aligned}
& \|f(x)-L(x)\| \leq n\|f(0)\|+\sum_{i=0}^{\infty} \frac{\varphi\left(n^{i} x, \cdots, n^{i} x\right)}{n^{i+1}} \\
& \left(\|f(x)-L(x)\| \leq \sum_{i=0}^{\infty} n^{i} \varphi\left(\frac{x}{n^{i+1}}, \cdots, \frac{x}{n^{i+1}}\right), \text { resp. }\right)
\end{aligned}
$$

for all $x \in{ }_{B} \mathbb{B}_{1}$.

Proof. By Theorem 3.1, it follows from the inequality of the statement for $a=1$ that there exists a unique additive mapping $L:{ }_{B} \mathbb{B}_{1} \rightarrow$ ${ }_{B} \mathbb{B}_{2}$ satisfying the inequality (3.1). Under the assumption that $f$ is measurable or $f(t x)$ is continuous in $t \in \mathbb{R}$ for each fixed $x \in{ }_{B} \mathbb{B}_{1}$, the mapping $L$ is $\mathbb{R}$-linear. And taking $x_{1}=\cdots=x_{n}:=x$ in (4.1), then we get

$$
\|n f(a x)-a f(n x)\| \leq n(n-1)\|f(0)\|+\varphi(x, x, \cdots, x)
$$

for all $x \in{ }_{B} \mathbb{B}_{1}$. Dividing (4.2) by $n^{m}$ and replacing $x:=n^{m-1} x(m \in \mathbb{N})$, we get $L(a x)=a L(x)$ for all $x \in{ }_{B} \mathbb{B}_{1}$ and all $a \in B(1)$ by taking $m \rightarrow \infty$. The last relation is trivially true for $a=0$. For each element $\alpha(\neq 0) \in B, \alpha=|\alpha| \cdot \frac{\alpha}{|\alpha|}$ and $\frac{\alpha}{|\alpha|} \in B(1)$. Since $L$ is $\mathbb{R}$-linear, we see

$$
L(\alpha x)=L\left(|\alpha| \cdot \frac{\alpha}{|\alpha|} x\right)=|\alpha| L\left(\frac{\alpha}{|\alpha|} x\right)=|\alpha| \cdot \frac{\alpha}{|\alpha|} L(x)=\alpha L(x)
$$

for each nonzero $\alpha \in B$ and all $x \in{ }_{B} \mathbb{B}_{1}$. So the unique $\mathbb{R}$-linear mapping $L$ is also $B$-linear, as desired.

Corollary 4.2. Let $p \neq 1$ be a positive real number and $\theta, \delta \geq 0$ be real numbers. If a mapping $f:{ }_{B} \mathbb{B}_{1} \rightarrow{ }_{B} \mathbb{B}_{2}$ satisfies the inequality

$$
\left\|D_{a} f\left(x_{1}, x_{2}, \cdots, x_{n}\right)\right\| \leq \delta+\theta \sum_{i=1}^{n}\left\|x_{i}\right\|^{p}
$$

for all $a \in B(1)$ and all $x_{1}, x_{2}, \cdots, x_{n} \in{ }_{B} \mathbb{B}_{1}$, where $\delta=0$ when $p>1$, and if $f$ is measurable or $f(t x)$ is continuous in $t \in \mathbb{R}$ for each fixed $x \in$ 
${ }_{B} \mathbb{B}_{1}$, then there exists a unique $B$-linear mapping $L:{ }_{B} \mathbb{B}_{1} \rightarrow{ }_{B} \mathbb{B}_{2}$ such that $L$ satisfies the inequality

$$
\|f(x)-L(x)\| \leq n\|f(0)\|+\frac{\delta}{n-1}+\frac{n^{p}}{\left|n-n^{p}\right|}\|x\|^{p}
$$

for all $x \in{ }_{B} \mathbb{B}_{1}$.

\section{References}

[1] J. Aczel, Lectures on Functional Equations and their Applications, Academic Press, New York, San Francisco, London, 1966.

[2] T. Aoki, On the stability of the linear transformation in Banach spaces, J. Math. Soc. Japan. 2 (1950), 64-66.

[3] Z. Gajda, On stability of additive mappings, Internat. J. Math. Math. Sci. 14 (1991), 431-434.

[4] P. Găvruta, A generalization of the Hyers-Ulam-Rassias stability of approximately additive mappings, J. Math. Anal. Appl. 184 (1994), 431-436.

[5] D. H. Hyers, On the stability of the linear functional equations, Proc. Natl. Acad. Sci. 27 (1941), 222-224.

[6] P. Nakmahachalasint, On the Hyers-Ulam-Rassias stability of an n-dimensional additive functional equation, Thai J. Math. 5 (2007), 81-86.

[7] Th. M. Rassias, On the stability of the linear mapping in Banach spaces, Proc. Amer. Math. Soc. 72 (1978), 297-300.

[8] S. M. Ulam, Problems in Modern Mathematics, Chapter 6, Wiley Interscience, New York, 1964.

Department of Mathematics

Chungnam National University

Daejeon 305-764, Republic of Korea

E-mail: hmkim@cnu.ac.kr

$* *$

Department of Mathematics

Chungnam National University

Daejeon 305-764, Republic of Korea

E-mail: hyshin31@cnu.ac.kr 